\title{
A Prospective Study of the Association of Metacognitive Beliefs and Processes with Persistent Emotional Distress After Diagnosis of Cancer
}

\author{
Sharon A. Cook • Peter Salmon • Graham Dunn • \\ Chris Holcombe $\cdot$ Philip Cornford • \\ Peter Fisher
}

Published online: 11 September 2014

(C) The Author(s) 2014. This article is published with open access at Springerlink.com

\begin{abstract}
Two hundred and six patients, diagnosed with primary breast or prostate cancer completed self-report questionnaires on two occasions: before treatment (T1) and 12 months later (T2). The questionnaires included: the Hospital Anxiety and Depression Scale; Impact of Events Scale; the Metacognitions Questionnaire-30 (MCQ-30) and the Illness Perceptions Questionnaire-revised. A series of regression analyses indicated that metacognitive beliefs at T1 predicted between 14 and $19 \%$ of the variance in symptoms of anxiety, depression and trauma at $\mathrm{T} 2$ after controlling for age and gender. For all three outcomes, the MCQ-30 subscale 'negative beliefs about worry' made the largest individual contribution with 'cognitive confidence' also contributing in each case. For anxiety, a third metacognitive variable, 'positive beliefs about worry' also predicted variance in $\mathrm{T} 2$ symptoms. In addition, hierarchical analyses indicated that metacognitive beliefs explained a small but significant amount of variance in $\mathrm{T} 2$ anxiety ( $2 \%$ ) and T2 depression ( $4 \%$ ) over and above that explained by demographic variables, $\mathrm{T} 1$ symptoms and $\mathrm{T} 1$ illness perceptions. The findings suggest that modifying metacognitive beliefs and processes has the potential to alleviate distress associated with cancer.
\end{abstract}

S. A. Cook $(\bowtie) \cdot$ P. Salmon · P. Fisher

Psychological Sciences, University of Liverpool, The Whelan

Building, Brownlow Hill, Liverpool L69 3GB, UK

e-mail: sacook@liv.ac.uk

S. A. Cook - P. Salmon - C. Holcombe · P. Cornford Royal Liverpool and Broadgreen University Hospitals NHS Trust, Liverpool, UK

G. Dunn

Centre for Biostatistics, The University of Manchester,

Manchester, UK
Keywords Metacognitive beliefs - Breast cancer . Prostate cancer - Emotional distress - Causal predictors

\section{Introduction}

Survival rates in cancer continue to improve. It is estimated that there are over 10 million cancer survivors in the USA (Institute of Medicine 2007), and around 2 million in the UK (Maddams et al. 2009). However, despite improved survival, cancer remains a life-threatening diagnosis which often has a profound emotional impact years after treatment has ended (Helgeson et al. 2004; Meyerowitz et al. 2008). Although emotional distress is considered a normal response around the time of cancer diagnosis, it is also common across the cancer trajectory with over a third of patients in treatment or long-term follow-up reporting clinically significant levels of distress, including anxiety and depression, that warrants intervention (Carlson et al. 2004), while life-time prevalence of cancer-related PTSD is $10-12 \%$ for breast cancer and $20 \%$ for other cancers (Andrykowski and Kangas 2010). In recognition of this continuing impact, health policies recommend that all patients should undergo psychological assessment at key point from diagnosis, and have prompt access to psychological support (Holland 1999; Institute of Medicine 2007; National Institute for Health and Clinical Excellence 2004).

A common and particularly influential approach to providing such support in cancer is cognitive behaviour therapy (CBT) (Watson et al. 2007; Williams and Dale 2006). It is based on the premise that negative illness appraisal (i.e. negative thoughts about cancer and its consequences) instigates and maintains distress. Research has indeed begun to show that negative thoughts about cancer 
are associated with current (Cook et al. 2014; Whitaker et al. 2008) or later distress (Llewellyn et al. 2007; Millar et al. 2005). However, negative thoughts are common, and especially so in people newly diagnosed with cancer, yet not everyone becomes distressed as a result of these thoughts. Furthermore, the focus of CBT on challenging negative thoughts is hard to reconcile with the clinical reality of an often uncertain future, and recent research has indicated that many patients, especially in the early stages of cancer treatment, find engaging with the negative content of their thoughts about cancer too difficult or distressing (Baker et al. 2012). Meta-analytic studies of CBT and other psychotherapeutic interventions in cancer have produced mixed results, with one recent meta-analysis concluding that small to moderate effect sizes are typical (Faller et al. 2013). Collectively these studies indicate that there is considerable room for improvement in psychotherapeutic effectiveness.

Focusing on understanding the mechanisms underlying the maintenance of emotional distress after cancer diagnosis may help to enhance the efficacy achieved by psychological interventions (Faller et al. 2013). Possible mechanisms of change are suggested by the metacognitive model of emotional disorder (Wells and Mathews 1994, 1996). This model asserts that it is not the negative content of thoughts about cancer that explains why distress is maintained but how the individual responds to those thoughts. For most people, periods of distress in relation to cancer, or any other stressor, are transitory. However, the metacognitive model proposes that people are vulnerable to persistent distress when they hold maladaptive metacognitive beliefs which guide them towards a particularly toxic style of sustained and inflexible conscious processing of negative thoughts and feelings about their cancer. This is called the cognitive attentional syndrome (CAS), and includes cognitive processes such as persistent worry and rumination, focussing of attention on threat, and maladaptive coping strategies (e.g. avoidance or thought suppression). Such processes ultimately 'backfire' by sustaining negative thinking and the sense of threat rather than allowing such experiences to fade naturally. According to this model, negative content of thoughts about cancer may trigger metacognitive beliefs and activate the CAS or may be a product of worry and rumination, but have no direct causal role in maintaining emotional distress. Modifying metacognitive beliefs and interrupting the CAS has been effective in treating depression and a range of anxiety disorders (see Wells 2009 for a review). In addition, metacognitive beliefs have been associated with heightened emotional distress in physical health populations including: Parkinson's disease (Allott et al. 2005), chronic fatigue (MaherEdwards et al. 2011), breast cancer (Cook et al. 2014;
Thewes et al. 2013) and prostate cancer (Cook et al. 2014).

Our previous research (Cook et al. 2014), indicated that metacognitive beliefs (specifically 'positive beliefs about worry' and 'negative beliefs about worry') were associated with concurrent symptoms of anxiety, depression and trauma among patients recently diagnosed with breast or prostate cancer, and that they explained additional variance in these outcomes after controlling for age, gender and negative content of thoughts about cancer (i.e. negative illness perceptions). Structural equation modelling found evidence consistent with the central predictions of the metacognitive model that these beliefs cause and maintain distress directly, but also indirectly by driving worry. These findings provide the first evidence consistent with the theory that metacognitive beliefs underlie emotional distress experienced by cancer patients. However in order to provide more compelling evidence of a causal role for metacognitive beliefs in maintaining emotional distress after cancer, prospective research is needed to demonstrate a temporal relationship. Consequently, the aim of this study is to explore whether metacognitive beliefs measured shortly after diagnosis (T1) predict symptoms of anxiety, depression and trauma 12 months later (T2) and to explore whether they add to the variance explained over and above previously implicated variables including $\mathrm{T} 1$ symptoms and content of thoughts about cancer (i.e. T1 illness perceptions). Specifically we hypothesised that:

(1) Metacognitive beliefs assessed around the time of diagnosis will prospectively predict variance in anxiety depression and trauma 12 months later

(2) Metacognitive beliefs assessed around the time of diagnosis will add to the variance explained in $\mathrm{T} 2$ anxiety, depression and trauma symptoms over and above demographic variables, $\mathrm{T} 1$ symptoms and $\mathrm{T} 1$ illness perceptions

\section{Methods}

Design

A prospective cohort design included a pre-treatment baseline and 12 month follow-up. The study was approved by the NHS North West 5 Research Ethics Committee (reference: 09/H1010/70).

Procedure

From February 2010 to May 2011, 229 patients were consecutively recruited through two pre-treatment cancer clinics at a National Health Service (NHS) teaching 
hospital in North-West England. Inclusion criteria were a diagnosis of primary breast or prostate cancer and at least 18 years of age. Patients with recurrent or metastatic disease and those judged by the clinical team or researcher to be too distressed or confused to give informed consent were excluded. T1 data was obtained from self-report questionnaires and medical records shortly after diagnosis before primary treatment. Follow-up data (T2) was collected 12 months later through self-report questionnaires mailed to participants' homes.

\section{Measures}

All Measures were Assessed Both at T1 and T2

The Hospital Anxiety and Depression Scale [HADS, (Zigmond and Snaith 1983)] assessed the primary outcomes of anxiety and depression. The HADS is the most widely used measure of anxiety and depression in physical illness and has been extensively validated for cancer populations (Luckett et al. 2010; Norton et al. 2013; Vodermaier and Millman 2011). It consists of fourteen items (seven in each subscale) scored on a 4-point scale. The subscale scores range from 0 to 21 , high scores indicating greater anxiety or depression and scores of 8 or more indicating clinically significant levels. In the current sample, both subscales had good internal consistency (Cronbach's $\alpha: .84 / .88$ for T1/T2 depression; .88/.89 for T1/T2 anxiety).

Trauma symptoms were assessed using the Impact of Events Scale [IES; (Horowitz et al. 1979)]. This 15-item measure yields a total score of $0-75$, with high scores indicating greater trauma. It has been validated as a screening measure of stress reactions after a range of traumatic events (Sundin and Horowitz 2002). There is no consensus on a cut-off score for clinically significant trauma. However, a score of 27 or more provided an overall correct classification rate of $80 \%$ in a large sample of motor vehicle accident survivors comprising both genders (Coffey et al. 2006), and has previously been used in cancer (Purnell et al. 2011). Internal consistency of the IES was excellent at both time points in the current sample (Cronbach's $\alpha$ : .90/.94).

The revised Illness Perceptions Questionnaire [IPQ-R (Moss-Morris et al. 2002)] was used to assess participants' appraisal of their cancer. It assessed thoughts, ideas and beliefs about cancer in several distinct areas, including: 'identity' (the number of symptoms participants attribute to their cancer); 'chronic timeline' (how long they think it will last); 'cyclical timeline' (the perception that symptoms are cyclical); 'consequences' (the perception that cancer has negative consequences on life); 'personal control' and 'treatment control' (the beliefs that it can be controlled or cured by their own actions or by treatment, respectively); 'illness coherence' (the extent to which they feel they understand their illness); 'emotional representation' (their emotional response to their illness); and beliefs about causality. As the IPQ-R was included to assess the relative importance of patients' illness appraisal, the emotional representation subscale was disregarded. The final section of the IPQ-R assesses patients' causal attributions. These items are typically not summed as a single scale but may be analysed as separate items or as groups devised on the basis of theory (Moss-Morris et al. 2002). As only psychological and/or behavioural attributions have contributed to variance in quality of life (Scharloo et al. 2010) or emotional distress (Kulik and Kronfeld 2005; Traeger et al. 2009) after diagnosis of cancer, the seven causal items which reflect these attributions (i.e. 'my own behaviour', 'my mental attitude', 'stress or worry', 'my emotional state', and 'my personality' 'family problems or worries' and 'overwork') were used to generate a single causal subscale ('psychological attributions') and the remaining causal items were disregarded. With the exception of the identity subscale (in which the items are dichotomous), all IPQ-R items are scored 1-5 with higher subscale scores indicating greater endorsement of that illness perception. In the current sample, Cronbach's $\alpha$ : of the IPQ-R subscales ranged from .64 ('personal control') to .82 ('chronic timeline'/ 'cyclical timeline') at time 1 and from .63 ('treatment control') to .89 ('cyclical timeline') at time 2, indicating relatively poor to good internal consistency.

Metacognitive beliefs were measured using the Metacognitions Questionnaire 30- [MCQ-30 (Wells and Cartwright-Hatton 2004)]. The MCQ-30 was developed specifically to assess key components of the metacognitive model of emotional disorder. It comprises five subscales: 'positive beliefs about worry'; 'negative beliefs about worry'; 'cognitive confidence'; 'need to control thoughts'; and 'cognitive self-consciousness'. For each, items are scored 1-4, yielding a total score of 6-24. High scores indicate more positive or negative beliefs about worry, reduced confidence in memory, greater belief in the need to control thoughts and an increased tendency towards selffocussed attention, respectively. In the current sample, Cronbach's $\alpha$ : of MCQ-30 subscales ranged from .73 ('need to control thoughts') to .89 ('positive beliefs about worry') at T1 and from .79 ('need to control thoughts') to .91 ('positive beliefs about worry') at T2, indicating adequate to excellent internal consistencey.

\section{Analysis}

The data were analysed using SPSS Version 20. As $<2 \%$ of the data were missing at the scale level, and these were confirmed to be missing completely at random, missing 
scores were imputed using the SPSS Expectation-Maximisation algorithm (Little and Rubin 1987).

As not all scales were normally distributed, nonparametric statistics (Mann-Whitney or Kruskal-Wallis) were used to compare participants who completed both assessments with those who only completed $\mathrm{T} 1$ assessment on age group (divided above and below the median age), gender, educational level, perceived emotional social support, stage of disease, T1 HADS and IES scores. They were also used to examine the relationship of each T2 outcome with demographic, clinical and social support variables. Where significant associations with $\mathrm{T} 2$ outcomes were found $(p<.05)$, the relevant variables were entered as demographic control variables in the first step of subsequent regression analyses.

Initially, the IPQ-R and MCQ-30 were analysed in parallel to identify which subscales within each measure independently predicted each T2 outcome. For the IPQ-R, hierarchical multiple regression analyses were first used to identify the $\mathrm{T} 1$ subscales associated with each $\mathrm{T} 2$ outcome (anxiety, depression and trauma) after controlling for demographic variables (Analysis 1 for each outcome). These analyses were then repeated, using just the significant IPQ-R subscales from Analysis 1, and also controlling for T1 symptoms of anxiety, depression or trauma (Analysis 2 for each outcome). As we had no a priori theory about which subscales would independently predict $\mathrm{T} 2$ outcomes, the IPQ-R subscales were included in each analysis using stepwise rather than forced entry. The subscales identified as independent predictors in Analysis 2 for each outcome were then entered as variables in Analysis 3 for that outcome (see below).

This sequence of analyses was repeated for the MCQ30 , thereby testing hypothesis 1 . We first identified the T1 MCQ-30 subscales that independently predict $\mathrm{T} 2$ outcomes after controlling for demographic variables (Analysis 1 for each outcome), and then entered these in a further analysis also controlling for $\mathrm{T} 1$ symptoms of anxiety, depression or trauma (Analysis 2 for each outcome). As with the IPQ-R analyses, as we had no a priori theory about which subscales would independently predict T2 outcomes, MCQ-30 subscales were included in each analysis using stepwise rather than forced entry. The subscales identified as independent predictors in Analysis 2 for each outcome were then entered as variables in Analysis 3 for that outcome (see below), which tested hypothesis 2 .

Final hierarchical multiple regression analyses (Analysis 3 for each outcome) assessed whether the T1 MCQ-30 subscales which had been identified as significant predictors in Analysis 2 (see above) were able to predict variance in $\mathrm{T} 2$ outcomes over and above that explained by demographic variables, $\mathrm{T} 1$ symptoms and the negative content of thoughts about cancer at Time 1 (i.e. IPQ-R subscales identified as significant predictors in Analysis 2). This final analysis used forced entry and bootstrapped sampling to ensure findings were robust.

\section{Results}

\section{Completers Versus Non Completers}

Of the 229 participants who completed T1 questionnaires $206(90 \%)$ also completed the assessment 12 months later. No significant differences between completers and noncompleters were apparent on T1 HADS and IES scores, age, gender, education, or tumour grade. However noncompleters were more likely than completers to report low levels of perceived emotional support at T1 (52 vs. $31 \%$ $p=.034)$.

\section{Sample Characteristics}

The baseline demographic and clinical characteristics of the final sample $(\mathrm{N}=206)$ are shown in Table 1. Women with breast cancer and younger patients were more anxious at $\mathrm{T} 2(\mathrm{U}=-3,269.5, p<.001, \mathrm{r}=-.27 ; \mathrm{U}=-3,721$, $p<.001 . \mathrm{r}=.26$ ), and reported more trauma symptoms $(\mathrm{U}=3,636, p=.003, \mathrm{r}=-.21 ; \mathrm{U}=3,638, p<.001$, $\mathrm{r}=.27)$ than did men with prostate cancer or older patients. Women with breast cancer also reported more symptoms of depression at $\mathrm{T} 2$ than did men with prostate cancer $(\mathrm{U}=3,857.5, p=.014, \mathrm{r}=.17)$. No outcome was related to education, perceived emotional support or tumour grade. Therefore just age and gender were used as demographic covariates in subsequent analyses. The levels of anxiety, depression and trauma symptoms at both time points are shown in Table 2. Both anxiety and trauma symptoms significantly declined over time, whereas depressive symptoms significantly increased.

Association of T1 Illness Perceptions with T2 Anxiety, Depression and Trauma

Regression of emotional distress on the IPQ-R subscales (Table 3) indicated that illness perceptions predicted between $10 \%$ (trauma) and $12 \%$ (anxiety) of the variance in T2 outcomes after controlling for age and gender (Analysis 1) and between $2 \%$ (trauma) and $3 \%$ (anxiety and depression) after also controlling for $\mathrm{T} 1$ symptoms (Analysis 2). The final models from Analysis 2 indicated that, perceived lack of personal control and negative perception of the consequences of cancer predicted $\mathrm{T} 2$ anxiety ( 1 and $2 \%$ respectively), while poor understanding of the illness ('illness coherence') predicted $\mathrm{T} 2$ depression and trauma. These IPQ- $\mathrm{R}$ subscales were therefore used to 
Table 1 Sample characteristics at time $1(\mathrm{~N}=206)$

N.B. Missing data: marital status $\mathrm{n}=5$; live alone $\mathrm{n}=3$; education $\mathrm{n}=9$; employment $\mathrm{n}=3$; tumour grade $\mathrm{n}=3$

control for content of thoughts about cancer in the final hierarchical multiple regression analyses.

Association of T1 Metacognitive Beliefs with T2 Anxiety, Depression and Trauma

The results of the hierarchical multiple regression analyses to test hypothesis 1 are shown in Table 4. After controlling for age and gender (Analysis 1), metacognitive beliefs explained an additional $19 \%$ of the variance in T2 anxiety, $15 \%$ of the variance in T2 depression and $14 \%$ of the variance in T2 trauma. In all cases 'negative beliefs about worry' made the largest individual contribution of all the predictors with 'cognitive confidence' also making a significant individual contribution. For anxiety, 'positive beliefs about worry' was a further significant individual predictor of T2 symptoms. After controlling for T1 symptoms as well as demographic variables (Analysis 2), metacognitive beliefs continued to predict a small but significant proportion of variance in each outcome. It added a significant $2 \%$ to the variance in T2 anxiety, $5 \%$ to the variance in $\mathrm{T} 2$ depression and $1 \%$ to the variance in $\mathrm{T} 2$ trauma. In each case, 'cognitive confidence' was the only MCQ-30 subscale that continued to make a significant individual contribution to the variance explained, and consequently this variable was the only metacognitive variable entered into the final set of analyses (Analysis 3).

Predictive ability of T1 Metacognitive Beliefs Over and Above: Demographic Variables, T1 Symptoms and Content of Thoughts about Cancer

The results of the hierarchical multiple regression analyses to test the second hypothesis (Analysis 3) are shown in Table 5. For anxiety and depression, 'cognitive confidence' added a significant 2 and $4 \%$ respectively to the variance in T2 symptoms over and above demographic variables, T1 symptoms and content of thoughts about cancer (i.e. relevant T1 illness perceptions). For anxiety, younger age, baseline symptoms, perceived lack of personal control and low cognitive confidence each made a significant individual contribution to the final model, which accounted for $42 \%$ of the variance in T2 symptoms. For depression, just younger age, baseline symptoms and low cognitive confidence made significant independent contributions to the final model, which accounted for $33 \%$ of the variance in T2 symptoms.

In the case of trauma, 'cognitive confidence' did not make any significant contribution to the variance explained in T2 symptoms after controlling for demographic variables, T1 symptoms and T1 illness perceptions ('illness coherence'). In fact, younger age and T1 symptoms were the only variables to make a significant individual contribution to the final model, which accounted for $39 \%$ of the variance.
Table 2 Distribution of anxiety, depression and trauma scores at both time-points

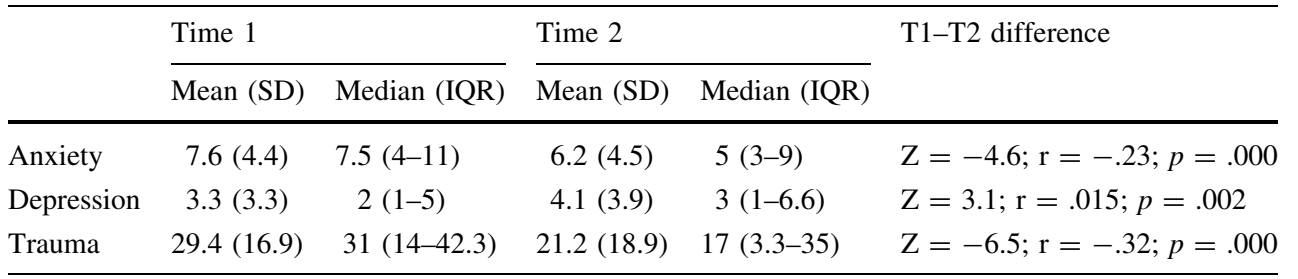


Table 3 Final models of the variance in T2 anxiety, depression and trauma predicted by T1 illness perceptions after controlling for age and gender (Analysis 1) and age, gender and T1 levels of symptoms (Analysis 2)

\begin{tabular}{|c|c|c|c|c|c|c|c|c|c|c|c|c|}
\hline & \multicolumn{4}{|l|}{$\mathrm{T} 2$ anxiety } & \multicolumn{4}{|c|}{$\mathrm{T} 2$ depression } & \multicolumn{4}{|l|}{$\mathrm{T} 2$ trauma } \\
\hline & $\mathrm{R}^{2}$ change & Beta & $\mathrm{T}$ & $p$ & $\mathrm{R}^{2}$ change & Beta & $\mathrm{T}$ & $p$ & $\mathrm{R}^{2}$ change & Beta & $\mathrm{T}$ & $p$ \\
\hline \multicolumn{13}{|l|}{ Analysis 1} \\
\hline Constant & & & 3.05 & .003 & & & 3.13 & .002 & & & 4.74 & .000 \\
\hline Step 1-demographics & $13 \%{ }^{* * *}$ & & & & $5 \%$ ** & & & & $14 \%^{* * *}$ & & & \\
\hline Gender & & -.19 & -3.04 & .003 & & -.13 & -1.94 & .054 & & -.14 & -2.28 & .023 \\
\hline Age & & -.21 & -3.28 & .001 & & -.11 & -1.62 & .106 & & -.27 & -4.19 & .000 \\
\hline Step 2-IPQ-R & $12 \% * * *$ & & & & $11 \%^{* *}$ & & & & $10 \%^{* * *}$ & & & \\
\hline Identity & & ns & ns & ns & & $\mathrm{ns}$ & ns & ns & & ns & ns & ns \\
\hline Cyclical timeline & & $\mathrm{ns}$ & ns & ns & & ns & ns & ns & & ns & ns & ns \\
\hline Chronic timeline & & ns & ns & ns & & ns & $\mathrm{ns}$ & $\mathrm{ns}$ & & ns & ns & ns \\
\hline Consequences & & .22 & 3.41 & .001 & & .19 & 2,75 & .006 & & .19 & 2.92 & .004 \\
\hline Illness coherence & & ns & ns & ns & & -.18 & -2.73 & .007 & & -.24 & -3.76 & .000 \\
\hline Psychological attributions & & .19 & 2.97 & .003 & & ns & ns & $\mathrm{ns}$ & & ns & ns & ns \\
\hline Personal control & & -.14 & -2.18 & .030 & & -.13 & -2.02 & .045 & & ns & ns & ns \\
\hline Treatment control & & ns & $\mathrm{ns}$ & $\mathrm{ns}$ & & ns & ns & ns & & ns & $\mathrm{ns}$ & $\mathrm{ns}$ \\
\hline $\mathrm{R}^{2}$ & $25 \%$ & & & & $16 \%$ & & & & $24 \%$ & & & \\
\hline $\operatorname{Adj} R^{2}$ & $23 \%$ & & & & $13 \%$ & & & & $23 \%$ & & & \\
\hline \multicolumn{13}{|l|}{ Analysis 2} \\
\hline Constant & & & 2.79 & .006 & & & 4.61 & .000 & & & 4.23 & .000 \\
\hline STEP 1-demographics & $13 \%{ }^{* * * *}$ & & & & $5 \%$ & & & & $14 \%{ }^{* * * *}$ & & & \\
\hline Gender & & -.03 & -.42 & .675 & & -.08 & -1.36 & .177 & & -.04 & -.65 & .516 \\
\hline Age & & -.17 & -2.99 & .003 & & -.15 & -2.41 & .017 & & -.22 & -3.69 & .000 \\
\hline Step 2-T1 symptoms & $25 \%$ *** & & & & $21 \%{ }^{* * *}$ & & & & $23 \%{ }^{* * * *}$ & & & \\
\hline T1 Anxiety & & .49 & 8.03 & .000 & & - & - & - & & - & - & - \\
\hline T1 depression & & - & - & - & & .44 & 7.25 & .000 & & - & - & - \\
\hline $\mathrm{T} 1$ trauma & & - & - & - & & - & - & - & & .46 & 7.40 & .000 \\
\hline Step 2-IPQ-R & $3 \%^{*}$ & & & & $3 \%{ }^{* *}$ & & & & $2 \%^{*}$ & & & \\
\hline Consequences & & .13 & 2.17 & .032 & & ns & ns & ns & & ns & ns & ns \\
\hline Illness coherence & & - & - & - & & -.16 & -2.65 & .009 & & -.13 & -2.23 & .025 \\
\hline Psychological attributions & & ns & $\mathrm{ns}$ & $\mathrm{ns}$ & & - & - & - & & - & - & - \\
\hline Personal control & & -.11 & -2.08 & .039 & & $\mathrm{~ns}$ & $\mathrm{~ns}$ & $\mathrm{~ns}$ & & - & - & - \\
\hline $\mathrm{R}^{2}$ & $41 \%$ & & & & $29 \%$ & & & & $39 \%$ & & & \\
\hline Adj $R^{2}$ & $39 \%$ & & & & $27 \%$ & & & & $37 \%$ & & & \\
\hline
\end{tabular}

$\mathrm{R}^{2}$ change shows increment in variance explained when each set of variables was entered sequentially; beta, $\mathrm{T}$ and $p$ are from the final model containing variables from all steps

N.B. IPQ-R subscales entered using stepwise method

$n s$ non significant, data not available using stepwise methods

$* p<.05, * * p<.01, * * * p<.001$

\#\# Only subscales found to be significant predictors in Analysis 1 were entered. - indicates variable not included

\section{Discussion}

This is the first study to explore whether metacognitive beliefs soon after cancer diagnosis, and before active treatment (T1), predict emotional distress 12 months later (T2). T1 metacognitive beliefs predicted T2 anxiety, depression, and trauma after controlling for age, gender and T1 symptoms, thus supporting hypothesis 1. This finding builds on previous research in non-clinical populations in which metacognitive beliefs prospectively predicted levels of anxiety and depression two (Weber and Exner 2013), three (Papageorgiou and Wells 2009; 
Table 4 Final models of the variance in T2 anxiety, depression and trauma predicted by T1 metacognitive beliefs after controlling for age and gender (Analysis 1) and age, gender and T1 levels of symptoms (Analysis 2)

\begin{tabular}{|c|c|c|c|c|c|c|c|c|c|c|c|c|}
\hline & \multicolumn{4}{|l|}{$\mathrm{T} 2$ anxiety } & \multicolumn{4}{|c|}{$\mathrm{T} 2$ depression } & \multicolumn{4}{|l|}{$\mathrm{T} 2$ trauma } \\
\hline & $\mathrm{R}^{2}$ change & Beta & $\mathrm{T}$ & $p$ & $\mathrm{R}^{2}$ change & Beta & $\mathrm{T}$ & $p$ & $\mathrm{R}^{2}$ change & Beta & $\mathrm{T}$ & $p$ \\
\hline \multicolumn{13}{|l|}{ Analysis 1} \\
\hline Constant & & & 2.98 & .003 & & & 1.19 & .236 & & & 3.64 & .000 \\
\hline Step 1-demographics & $13 \%^{* * *}$ & & & & $5 \%{ }^{* *}$ & & & & $14 \%^{* * *}$ & & & \\
\hline Gender & & -.16 & -2.67 & .008 & & -.10 & -1.60 & .112 & & -.11 & -1.70 & .091 \\
\hline Age & & -20 & -3.39 & .001 & & -.10 & -1.62 & .107 & & -.26 & -4.24 & .000 \\
\hline Step 2-MCQ-30 & $19 \%$ & & & & $15 \%^{* * *}$ & & & & $14 \%^{* * * *}$ & & & \\
\hline POS & & .17 & 2.58 & .011 & & ns & ns & ns & & ns & ns & ns \\
\hline NEG & & .28 & 3.90 & .000 & & .25 & 3.64 & .000 & & .28 & 4.31 & .000 \\
\hline $\mathrm{CC}$ & & .12 & 2.00 & .047 & & .22 & 3.34 & .001 & & .17 & 2.66 & .008 \\
\hline $\mathrm{NC}$ & & ns & ns & ns & & ns & ns & ns & & ns & ns & ns \\
\hline $\mathrm{CSC}$ & & ns & ns & ns & & ns & ns & ns & & ns & ns & ns \\
\hline $\mathrm{R}^{2}$ & $32 \%$ & & & & $20 \%$ & & & & $27 \%$ & & & \\
\hline Adj $R^{2}$ & $30 \%$ & & & & $18 \%$ & & & & $26 \%$ & & & \\
\hline \multicolumn{13}{|l|}{ Analysis 2} \\
\hline Constant & & & 3.70 & .000 & & & 2.34 & .020 & & & 3.19 & .002 \\
\hline Step 1-demographics & $13 \%^{* * * *}$ & & & & $5 \%$ ** & & & & $14 \%^{* * *}$ & & & \\
\hline Gender & & -.03 & -.54 & .590 & & -.09 & -1.42 & .158 & & -.04 & -.62 & .537 \\
\hline Age & & -.20 & -3.62 & .000 & & -.13 & -2.18 & .030 & & -.21 & -3.51 & .001 \\
\hline Step 2-T1 symptoms & $25 \% \%^{* * *}$ & & & & $21 \%^{* * *}$ & & & & $23 \%^{* * * *}$ & & & \\
\hline $\mathrm{T} 1$ anxiety & & .50 & 8.22 & .000 & & - & - & - & & - & - & - \\
\hline $\mathrm{T} 1$ depression & & - & - & - & & .42 & 7.06 & .000 & & - & - & - \\
\hline $\mathrm{T} 1$ trauma & & - & - & - & & - & - & - & & .47 & 7.44 & .000 \\
\hline Step $3-M C Q-30^{\# \#}$ & $2 \%^{*}$ & & & & $5 \%^{* * * *}$ & & & & $1 \%{ }^{*}$ & & & \\
\hline POS & & ns & ns & ns & & - & - & - & & - & - & - \\
\hline NEG & & $\mathrm{ns}$ & ns & $\mathrm{ns}$ & & ns & ns & ns & ns & ns & ns & ns \\
\hline $\mathrm{CC}$ & & .14 & 2.53 & .012 & & .23 & 3.85 & .000 & & .13 & 2.16 & .032 \\
\hline $\mathrm{R}^{2}$ & $40 \%$ & & & & $31 \%$ & & & & $38 \%$ & & & \\
\hline $\operatorname{Adj} R^{2}$ & $39 \%$ & & & & $30 \%$ & & & & $36 \%$ & & & \\
\hline
\end{tabular}

$\mathrm{R}^{2}$ change shows increment in variance explained when each set of variables was entered sequentially; beta, $\mathrm{T}$ and $p$ are from the final model containing variables from all steps

N.B. MCQ-30 subscales entered using stepwise method. MCQ-30 subscales: positive beliefs about worry (POS); negative beliefs about the danger and uncontrollability of worry (NEG); cognitive confidence (CC); need for control (NC); cognitive self-consciousness (CSC)

$n s$ non significant, data not available using stepwise methods

$* p<.05, * * p<.01, * * * p<.001$

\#\# Only subscales found to be significant predictors in Analysis 1 were entered. - indicates variable not included

Hjemdal et al. 2013) and 6 months (Yilmaz et al. 2011) later, after controlling for age, gender and T1 levels of symptoms.

Before controlling for $\mathrm{T} 1$ symptoms, metacognitive beliefs explained a greater proportion of variance in $\mathrm{T} 2$ anxiety, depression and trauma than did illness perceptions. Furthermore the illness perception subscales that were predictive ('consequences', 'personal control', 'psychological attributions', 'illness coherence') could be considered to be markers for worry or rumination in that they may be the outcome of these processes. Of the five MCQ-30 subscales included in Analysis 1, two ('negative beliefs about worry' and 'cognitive confidence') independently predicted T2 anxiety, depression and trauma, with a third ('positive beliefs about worry') also significantly contributing to the variance in anxiety. In all three cases 'negative beliefs about worry' made the largest individual contribution, as would be predicted by the metacognitive model of emotional disorder (Wells 2009). These findings are also consistent with those of (Yilmaz et al. 2011) who reported that, in their non-clinical sample, 'negative beliefs about worry' predicted levels of anxiety and depression 6 months 
Table 5 Final models of the variance in T2 anxiety, depression and trauma predicted by T1 metacognitive beliefs after controlling for age, gender, T1 level of symptoms and T1 illness perceptions (Analysis 3)

\begin{tabular}{|c|c|c|c|c|c|c|c|c|c|c|c|c|}
\hline & \multicolumn{4}{|l|}{$\mathrm{T} 2$ anxiety } & \multicolumn{4}{|c|}{$\mathrm{T} 2$ depression } & \multicolumn{4}{|l|}{$\mathrm{T} 2$ trauma } \\
\hline & $\mathrm{R}^{2}$ change & Beta & $\mathrm{T}$ & $p$ & $\mathrm{R}^{2}$ change & Beta & $\mathrm{T}$ & $p$ & $\mathrm{R}^{2}$ change & Beta & $\mathrm{T}$ & $p$ \\
\hline \multicolumn{13}{|l|}{ Analysis 3} \\
\hline Constant & & & 2.50 & .013 & & & 2.98 & .003 & & & 3.66 & .000 \\
\hline Step 1-demographics & $13 \%^{* * * *}$ & & & & $5 \%{ }^{* *}$ & & & & $14 \%^{* * *}$ & & & \\
\hline Gender & & -.04 & -.63 & .527 & & -.09 & -1.50 & .136 & & -.05 & -.79 & .430 \\
\hline Age & & -.18 & -3.06 & .003 & & -.14 & -2.29 & .023 & & -.22 & -3.73 & \\
\hline Step 2-T1 symptoms & $25 \%$ *** & & & & $21 \%^{* * * *}$ & & & & $23 \%^{* * * *}$ & & & \\
\hline T1 anxiety & & .46 & 7.49 & .000 & & - & - & - & & - & - & - \\
\hline T1 Depression & & - & - & - & & .41 & 6.86 & .000 & & - & - & - \\
\hline $\mathrm{T} 1$ trauma & & - & - & - & & - & - & - & & .44 & 6.77 & .000 \\
\hline Step 3-IPQ-R $R^{\# \#}$ & $3 \%$ ** & & & & $3 \%$ ** & & & & $2 \%^{*}$ & & & \\
\hline Consequences & & .12 & 1.94 & .054 & & - & - & - & & - & - & - \\
\hline Illness coherence & & - & - & - & & -.11 & -1.83 & .068 & & -.11 & -1.90 & .058 \\
\hline Personal control & & -.11 & -2.09 & .038 & & - & - & - & & - & - & - \\
\hline Step 4-MCQ-30"\# & $2 \%^{*}$ & & & & $4 \%^{* *}$ & & & & $1 \%{ }^{\mathrm{ns}}$ & & & \\
\hline $\mathrm{CC}$ & & .13 & 2.32 & .02 & & .20 & 3.31 & .001 & & .11 & 1.78 & .076 \\
\hline \multicolumn{13}{|l|}{ Model summary } \\
\hline $\mathrm{R}^{2}$ & $42 \%$ & & & & $33 \%$ & & & & $39 \%$ & & & \\
\hline Adj $R^{2}$ & $41 \%$ & & & & $31 \%$ & & & & $37 \%$ & & & \\
\hline
\end{tabular}

$\mathrm{R}^{2}$ change shows increment in variance explained by each step; beta, $\mathrm{T}$ and $p$ are from the final model containing variables from all steps

N.B. All variables entered using forced entry method. MCQ-30 subscales: cognitive confidence (CC)

\#\# Only subscales found to be significant predictors in Analysis 2 were entered. - indicates variable not included

$* p<.05, * * p<.01, * * * p<.001$

later. However, in the current study when T1 levels of distress were controlled, the relationship of 'negative beliefs about worry' with anxiety and depression was no longer significant. Instead 'cognitive confidence' was the only metacognitive variable to contribute to variance. The reasons for this are not clear.

One possibility is that this finding is due to the limitations of using hierarchical regression in a prospective study design where baseline emotional distress inevitably predominates in predicting future distress. Previous research (Cook et al. 2014) has demonstrated a strong cross-sectional association of $\mathrm{T} 1$ symptoms of anxiety, depression and trauma with metacognitive beliefs and processes. Consequently, as the metacognitive beliefs and processes that we measured to predict $\mathrm{T} 2$ distress also (according to theory) cause $\mathrm{T} 1$ distress, there is likely to be considerable overlap in the variance in $\mathrm{T} 2$ distress explained by $\mathrm{T} 1$ symptoms and metacognitive beliefs, leading to underestimation of the importance of the putative causal variables. That is, by controlling for baseline symptoms we may be masking the effect of the beliefs and processes that underlie its maintenance. To resolve this dilemma, approaches to analysis are required that can distinguish putatively causal effects arising from metacognitive beliefs and processes (causing symptoms of distress to be maintained) from the confounding effect resulting from symptom maintenance. Such differentiation is not feasible using standard hierarchical regression but may be possible using structural equation modelling techniques to model the effect of change in metacognitive beliefs on change in emotional distress.

As well as being able to explain more of the variance in T2 distress than did illness perceptions in Analysis 1, metacognitive beliefs ('cognitive confidence') were also able to explain additional variance in anxiety and depression over and above age, gender, T1 symptoms, and T1 illness perceptions (Analysis 3). This supports hypothesis 2 for these two outcomes. However, for trauma, metacognitive beliefs ('cognitive confidence') no longer significantly predicted T2 symptoms after including T1 illness perceptions ('illness coherence') in the analysis (Analysis 3). However, it should be noted that the proportion of variance in T2 trauma explained by 'cognitive confidence' is unchanged between Analysis 2 (controlling for T1 trauma) and Analysis 3 (controlling for T1 trauma and T1 'illness coherence'). Furthermore, there is little difference in the 
variance explained by 'cognitive confidence' in trauma $(1 \%)$ and in anxiety $(2 \%)$. Therefore this apparent discrepancy may be an artefact of the present data. To our knowledge this is the first study to explore the prospective relationship between metacognitive beliefs (as measured by the MCQ-30) and trauma symptoms, making it difficult to judge the reliability of this finding.

One limitation of the study is the restriction of the sample to breast and prostate cancer patients. These populations were selected because they represent the largest tumour groups in each gender and have a broadly similar prognosis. However this means it is not possible, in this sample, to separate out any effects that may be due to tumour group or gender. Furthermore, we cannot assume that the predictive effects found in this study would generalise to other cancer populations. Further studies will be needed to test the stability of the observed predictive effect of metacognitive beliefs on persistent emotional distress across genders and different tumour and prognostic groups. In addition, despite the prospective design, it should be noted that causality can still not be assumed as the influence of unmeasured confounders cannot be ruled out. In order to provide more compelling evidence of a causal role for metacognitive beliefs, further studies are necessary that adopt different approaches to design, such as experimental manipulation.

In summary, the findings of the current study provide promising first evidence that metacognitive beliefs can help to predict anxiety, depression and trauma 1 year after diagnosis of breast and prostate cancer. Furthermore they support the hypothesis that metacognitive beliefs add to the variance explained in persistent anxiety and depression over and above that explained by negative content of thoughts about cancer. Consequently, therapeutic approaches targeting metacognitive beliefs and processes, rather than the content of negative thoughts about cancer, may prove beneficial for preventing persistent emotional distress in these populations.

Acknowledgments This research was conducted as part of a Population Health Scientist Fellowship funded by the Medical Research Council. We gratefully thank the staff and patients of the Royal Liverpool and Broadgreen University NHS Hospital Trust for supporting this study and also Kirsten Atherton, Helen Ullmer and Chris Huntley for their contributions to the data acquisition.

Conflict of Interest Sharon A. Cook, Peter Salmon, Graham Dunn, Chris Holcombe, Philip Cornford and Peter Fisher declare that they have no conflict of interest.

Informed Consent All procedures followed were in accordance with the ethical standard of the responsible committee on human experimentation (institutional and national) and with the Helsinki Declaration of 1975, as revised in 2000 (5). The study was approved by the NHS North West 5 Research Ethics Committee (reference:
09/H1010/70). Informed consent was obtained from all patients for being included in the study.

Animal Rights No animal studies were carried out by the authors for this article.

Open Access This article is distributed under the terms of the Creative Commons Attribution License which permits any use, distribution, and reproduction in any medium, provided the original author(s) and the source are credited.

\section{References}

Allott, R., Wells, A., Morrison, A. P., \& Walker, R. (2005). Distress in Parkinson's disease: Contributions of disease factors and metacognitive style. British Journal of Psychiatry, 187, 182-183. doi:10.1192/bjp.187.2.182.

Andrykowski, M. A., \& Kangas, M. (2010). Posttraumatic stress disorder associated with cancer diagnosis and treatment. In J. C. Holland, W. Breitbart, P. B. Jacobsen, M. S. Lederberg, M. J. Loscalzo, \& R. McCorkle (Eds.), Psycho-Oncology (pp. 348-357). New York: Oxford University Press Inc.

Baker, P., Beesley, H., Dinwoodie, R., Fletcher, I., Ablett, J., Holcombe, C., et al. (2012). 'You're putting thoughts into my head': A qualitative study of the readiness of patients with breast, lung or prostate cancer to address emotional needs through the first 18 months after diagnosis. Psychooncology,. doi:10.1002/pon.3156.

Carlson, L. E., Agen, M., Cullum, J., Goodey, E., Koopmans, J., Lamont, L., et al. (2004). High Levels of untreated distress and fatigue in cancer patients. British Journal of Cancer, 90, 2297-2304. doi:10.1038/sj.bjc.660188.

Coffey, S. F., Gudmundsdottir, B., Beck, J. G., Palyo, S. A., \& Miller, L. (2006). Screening for PTSD in motor vehicle accident survivors using the PSS-SR and IES. Journal of Traumatic Stress, 19(1), 119-128. doi:10.1002/jts.20106.

Cook, S. A., Salmon, P., Holcombe, C., Cornford, P., Dunn, G., \& Fisher, P. (2014). The association of metacognitive beliefs with emotional distress after diagnosis of cancer. Health Psychology. doi:10.1037/hea0000096.

Faller, H., Schuler, M., Richard, M., Heckl, U., Weis, J., \& Kuffner, R. (2013). Effects of psycho-oncologic interventions on emotional distress and quality of life in adult patients with cancer: Systematic review and meta-analysis. Journal of Clinical Oncology, 31(6), 782-793. doi:10.1200/JCO.2011.40.8922.

Helgeson, V. S., Snyder, P., \& Seltman, H. (2004). Psychological and physical adjustment to breast cancer over 4 years: Identifying distinct trajectories of change. Health Psychology, 23(1), 3-15. doi:10.1037/0278-6133.23.1.3.

Hjemdal, O., Stiles, T., \& Wells, A. (2013). Automatic thoughts and meta-cognition as predictors of depressive or anxious symptoms: A prospective study of two trajectories. Scandinavian Journal of Psychology, 54(2), 59-65. doi:10.1111/sjop.12010.

Holland, J. C. (1999). Update: NCCN practice guidelines for the management of psychosocial distress. Oncology, Suppl, 13, 459-507.

Horowitz, M., Wilner, N., \& Alvarez, W. (1979). Impact of event scale: A measure of subjective stress. Psychosomatic Medicine, 41(3), 209-218.

Institute of Medicine. (2007). Cancer care for the whole patient: meeting psychosocial health needs. Washington, DC: The National Academies Press.

Kulik, L., \& Kronfeld, M. (2005). Adjustment to breast cancer: The contribution of resources and causal attributions regarding the 
illness. Social Work in Health Care, 41(2), 37-57. doi:10.1300/ J010v41n02_03.

Little, R. J. A., \& Rubin, D. B. (1987). Statistical analysis with missing data. New York: Wiley.

Llewellyn, C. D., McGurk, M., \& Weinman, J. (2007). Illness and treatment beliefs in head and neck cancer: Is Leventhal's common sense model a useful framework for determining changes in outcomes over time? Journal of Psychosomatic Research, 63(1), 17-26. doi:10.1016/j.jpsychores.2007.01.013.

Luckett, T., Butow, P. N., King, M. T., Oguchi, M., Heading, G., Hackl, N. A., et al. (2010). A review and recommendations for optimal outcome measures of anxiety, depression and general distress in studies evaluating psychosocial interventions for English-speaking adults with heterogeneous cancer diagnoses. Supportive Care in Cancer, 18(10), 1241-1262. doi:10.1007/ s00520-010-0932-8.

Maddams, J., Brewster, D., Gavin, A., Steward, J., Elliott, J., Utley, M., et al. (2009). Cancer prevalence in the United Kingdom: Estimates for 2008. British Journal of Cancer, 101(3), 541-547. doi:10.1038/sj.bjc.6605148.

Maher-Edwards, L., Fernie, B. A., Murphy, G., Nikcevic, A. V., \& Spada, M. M. (2011). Metacognitive factors in chronic fatigue syndrome. Clinical Psychology and Psychotherapy,. doi:10. 1002/cpp.757.

Meyerowitz, B. E., Kurita, K., \& D'Orazio, L. M. (2008). The Psychological and Emotional Fallout of Cancer and Its Treatment. Cancer Journal, 14(6), 410-413.

Millar, K., Purushotham, A. D., McLatchie, E., George, W. D., \& Murray, G. D. (2005). A 1-year prospective study of individual variation in distress, and illness perceptions, after treatment for breast cancer. Journal of Psychosomatic Research, 58(4), 335-342. doi:10.1016/j.jpsychores.2004.10.005.

Moss-Morris, R., Weinman, J., Petrie, K. J., Horne, R., Cameron, L. D., \& Buick, D. (2002). The revised illness perception questionnaire (IPQ-R). Psychology and Health, 17, 1-16.

National Institute for Health and Clinical Excellence. (2004). Improving supportive and palliative care for adults with cancer: The manual. London: NICE.

Norton, S., Cosco, T., Doyle, F., Done, J., \& Sacker, A. (2013). The Hospital Anxiety and Depression Scale: A meta confirmatory factor analysis. Journal of Psychosomatic Research, 74(1), 74-81. doi:10.1016/j.jpsychores.2012.10.010.

Papageorgiou, C., \& Wells, A. (2009). A prospective test of the clinical metacognitive model of rumination and depression. International Journal of Cognitive Therapy, 2(2), 123-131. doi:10.1521/ijct.2009.2.2.123.

Purnell, J. Q., Palesh, O. G., Heckler, C. E., Adams, M. J., Chin, N., Mohile, S., et al. (2011). Racial disparities in traumatic stress in prostate cancer patients: Secondary analysis of a National URCC CCOP Study of 317 men. Supportive Care in Cancer, 19(7), 899-907. doi:10.1007/s00520-010-0880-3.

Scharloo, M., de Jong, R. J. B., Langeveld, T. P. M., van VelzenVerkaik, E., den Akker, M. M. D. O., \& Kaptein, A. A. (2010).
Illness cognitions in head and neck squamous cell carcinoma: Predicting quality of life outcome. Supportive Care in Cancer, 18(9), 1137-1145. doi:10.1007/s00520-009-0728-x.

Sundin, E. C., \& Horowitz, M. J. (2002). Impact of Event Scale: Psychometric properties. British Journal of Psychiatry, 180, 205-209. doi:10.1192/bjp.180.3.205.

Thewes, B., Bell, M. L., \& Butow, P. (2013). Fear of cancer recurrence in young early-stage breast cancer survivors: The role of metacognitive style and disease-related factors. Psychooncology, 22(9), 2059-2063. doi:10.1002/pon.3252.

Traeger, L., Penedo, F. J., Gonzalez, J. S., Dahn, J. R., Lechner, S. C., Schneiderman, N., et al. (2009). Illness perceptions and emotional well-being in men treated for localized prostate cancer. Journal of Psychosomatic Research, 67(5), 389-397. doi:10.1016/j.jpsychores.2009.03.013.

Vodermaier, A., \& Millman, R. D. (2011). Accuracy of the Hospital Anxiety and Depression Scale as a screening tool in cancer patients: A systematic review and meta-analysis. Supportive Care in Cancer, 19(12), 1899-1908. doi:10.1007/s00520-0111251-4.

Watson, M., White, C., Ashley, S., Davolls, S., \& Hopwood, P. (2007). Problem focused interactive telephone therapy (ProFITT (c)) for cancer patients: A feasibility study. Psychooncology, 16(9), 128.

Wells, A. (2009). Metacogntive therapy for anxiety and depression. New York: NY Guilford Press.

Wells, A., \& Cartwright-Hatton, S. (2004). A short form of the Metacognitions Questionnaire: Properties of the MCQ-30. Behaviour Research and Therapy, 42(4), 385-396. doi:10. 1016/S0005-7967(03)00147-5.

Wells, A., \& Mathews, G. (1994). Attention and Emotion. A clinical perspective. Hove: Erlbaum.

Wells, A., \& Mathews, G. (1996). Modelling cognition in emotional disorder: the S-REF model. Behaviour Research and Therapy, 32, 867-870. doi:10.1016/0005-7967(94)90167-8.

Whitaker, K. L., Brewin, C. R., \& Watson, M. (2008). Intrusive cognitions and anxiety in cancer patients. Journal of Psychosomatic Research, 64(5), 509-517. doi:10.1016/j.jpsychores.2008. 02.009 .

Williams, S., \& Dale, J. (2006). The effectiveness of treatment for depression/depressive symptoms in adults with cancer: A systematic review. British Journal of Cancer, 94(3), 372-390. doi:10.1038/sj.bjc.6602949.

Yilmaz, A. E., Gencoz, T., \& Wells, A. (2011). The temporal precedence of metacognition in the development of anxiety and depression symptoms in the context of life-stress: A prospective study. Journal of Anxiety Disorders, 25(3), 389-396. doi:10. 1016/j.janxdis.2010.11.001.

Zigmond, A. S., \& Snaith, R. P. (1983). The hospital anxiety and depression scale. Acta Psychiatrica Scandinavica, 67(6), 361-370. doi:10.1111/j.1600-0447.1983.tb09716.x. 